\title{
The connection between mass loss and nucleosynthesis
}

\author{
Jacco Th. van Loon \\ Lennard-Jones Laboratories, Keele University, Staffordshire ST5 5BG, United Kingdom
}

\begin{abstract}
I discuss the relationship between mass loss and nucleosynthesis on the Asymptotic Giant Branch (AGB). Because of thermal pulses and possibly other mixing processes, products of nucleosynthesis can be brought to the surface of AGB stars, increasingly so as the star becomes more luminous, cooler, and unstable against pulsation of its tenuous mantle. As a result, mass loss is at its most extreme when dredge-up is too. As the high rate of mass loss truncates AGB evolution, it determines the enrichment of interstellar space with the AGB nucleosynthesis products. The changing composition of the stellar atmosphere also affects the mass-loss process, most obviously in the formation of dust grains - which play an important rôle in driving the wind of AGB stars.
\end{abstract}

Keywords: Molecular and chemical processes and interactions, Dust processes, Stellar structure, interiors, evolution, and nucleosynthesis, Stellar atmospheres, Circumstellar shells and expanding envelopes, Mass loss and stellar winds, Pulsations, Abundances and chemical composition, Giant stars, Carbon stars, S stars, and related types, Long-period variables (Miras) and semiregulars

PACS: 95.30.Ft, 95.30.Wi, 97.10.Cv, 97.10.Ex, 97.10.Fy, 97.10.Me, 97.10.Sj, 97.10.Tk, 97.20.Li, 97.30.Hk, 97.30.Jm

\section{INTRODUCTION}

Asymptotic Giant Branch (AGB) stars span a range in masses from $<1 \mathrm{M}_{\odot}$ to $\sim 8 \mathrm{M}_{\odot}$, which makes them important diagnostics of the star formation history and important players in galactic chemical enrichment from ages as young as $<100 \mathrm{Myr}$ up to (nearly) a Hubble time. They shed much, often most, of their initial mass in the form of dusty winds before leaving a white dwarf behind.

In this review I will first ask the question why we care about mass loss, with a bias towards those amongst us who are interested in nucleosynthesis. I then briefly discuss what determines the mass-loss rate, and comment on ways in which nucleosynthesis may, or may not, alter the conditions for the mass-loss mechanism.

\section{WHY DO WE CARE ABOUT MASS LOSS?}

Besides its importance for galactic recycling processes and the build-up of chemical richesse, mass loss greatly affects the star itself. Although mass loss may be seen as an outer boundary condition, it does affect the stellar interior.

As a result of mass loss the convective mantle reduces in mass as well as density. This enhances the effects from $3^{\text {rd }}$ dredge-up, as a given mass of dredged-up product of nucleosynthesis is going to lead to a larger enrichment of the mantle (and photosphere) if it is less diluted by pre-existing material. Massive AGB stars (roughly $M_{\text {init }}>4$ 
$\mathrm{M}_{\odot}$ ) experience Hot Bottom Burning (HBB), which converts carbon into nitrogen (and oxygen), which is the reason why they do not normally become carbon stars. However, if mass loss depletes the mantle to near extinction, HBB ceases and a final thermal pulse will almost certainly turn the star into a carbon star.

Once the mantle mass is depleted to the extent that it becomes transparent, the star leaves the AGB and gradually dies. The higher the rate of mass loss on the AGB, the earlier this happens and the fewer thermal pulses the AGB stars will have experienced. This affects the photospheric abundances observed near the tip of the AGB, as well as the chemical and isotopic yields from AGB stars. Mass loss will also truncate the growth of the core, and thus affect the mass distribution of white dwarfs.

Finally, as the convective envelope expands as a reaction to its diminished density, the surface gravity and temperature drop, which would appear to facilitate further mass loss. Stars for which this becomes important are generally seen to pulsate radially in the fundamental mode, and their pulsation period increases with increasing radius. If the radius increases to the extent that the pulsation period approaches the thermal timescale then the pulsating mantle will continuously adapt its thermal structure as it expands, and this might detach the mantle to form a planetary nebula $(\mathrm{PN})$.

The observed enrichment of the interstellar medium and subsequent generations of stars which is due to AGB stars depends on the AGB mass loss. This is true for elements as well as isotopic ratios; [12], for example, show that the [34] mass-loss formalism leads to a $75 \%$ reduction in the yields of elements like silicon and aluminium compared to using the Reimers' Law [21].

The dust production by AGB stars is also affected by the mass-loss history, but in a slightly different way [4]. We shall later see how the dust depends on the detailed chemical composition of the star, but it is already obvious that carbon stars would form very different dust from other, oxygen-rich AGB stars. Depending on how the mass loss varies in time, this may be accompanied by more or less dust mass, and more or less of the dust produced whilst the star is a carbon star (if it ever becomes one). This may all depend on initial metallicity; [4] predict that metal-poor AGB stars produce predominantly carbonaceous dust also if they are massive.

The Initial-Final Mass (IFM) relation depends on how quickly the mass loss truncates the growth of the core on the AGB. Measurements of the IFM relation in galactic open clusters [37] and central stars of PNe in the metal-poor Magellanic Clouds [35] suggest that it varies very little with metallicity. As a consequence, the total amount of mass shed on the AGB is quite well known, e.g., $\Delta M=4.1 \pm 0.1 \mathrm{M}_{\odot}$ for $M_{\text {init }}=5 \mathrm{M}_{\odot}$. This is because mass loss $\left(\dot{M} \gg 10^{-7} \mathrm{M}_{\odot} \mathrm{yr}^{-1}\right)$ outpaces nuclear burning for long enough ( $\sim 0.1-1 \mathrm{Myr}$ ), causing negligible core growth whilst the mantle is shed. This is true for the solar neighbourhood, with $Z_{\text {init }}=\mathrm{Z}_{\odot}$, but also in the $\mathrm{LMC}, Z_{\text {init }}=0.3-0.5 \mathrm{Z}_{\odot}$, and $\mathrm{SMC}, Z_{\text {init }}=0.1-0.2 \mathrm{Z}_{\odot}[30,26]$ and other metal-poor dwarf galaxies [8, 8, 14], and even if mass-loss rates were reduced by an order of magnitude ${ }^{1}$.

For low-mass AGB stars the preceding first ascent red giant branch (RGB) phase also involves substantial mass loss. This affects the mantle mass with which the star ascends the AGB, and thus its AGB evolution and mass loss. It has been suggested recently

\footnotetext{
${ }^{1}$ Note that this is not the case for red supergiants, which therefore explode as core-collapse supernovae.
} 
[6, 11] that super-solar metal-rich RGB stars may lose mass at a higher rate, truncating their RGB evolution, evading the helium flash and producing low-mass white dwarfs. These stars would never become AGB stars. Note that only a doubling of the mass lost on the RGB is required for this to happen. RGB mass loss seems to be fine-tuned within a narrow range though, as even in very metal-poor globular clusters it is inferred to explain the blue horizontal branches whilst still having to allow for the observed AGB stars and a PN in M 15, Z $=0.005 \mathrm{Z}_{\odot}$ (cf. [33]).

\section{WHAT DETERMINES THE MASS-LOSS RATE?}

Several mechanisms that could, in principle, drive mass loss do not work in AGB stars. The main and efficient driver of hot star winds, radiation pressure in atomic and ionic transitions does not work in AGB stars because they are not hot enough to produce enough opacity and not luminous enough to provide enough pressure. AGB stars do not reach the Eddington luminosity as defined in terms of the radiation pressure in their optically thick mantles, nor do they rotate fast enough to initiate or significantly facilitate mass loss. Radiation pressure in molecular bands is not thought to be sufficient to support a massive outflow either: because the bands are broad and the wind will be slow, the wind never accelerates out of its own shadow, and therefore only utilises the minor fraction of the luminosity that is emitted within the molecular bands.

What does work, at least in some cases, is the two-stage mechanism of pulsationinduced levitation, and radiation pressure on dust forming in this cool, dense molecular atmosphere. The pulsation is the result of oscillations in the partial ionization zône of hydrogen within the mantle, as the ionization stage determines the opacity in the mantle and thus the balance between gravity and the outward-directed radiation pressure, the so-called $\kappa$-mechanism. The pulsation is stronger at lower temperature and higher luminosity. Dust forms preferentially in the dense shocks during part of this pulsation cycle (which has a timescale of $10^{2-3}$ days), and contributes to driving a wind via radiation pressure and collisional coupling with the gas [1, 36].

The dust-driven wind regimes in the Hertzsprung-Russell diagram are set by the Eddington luminosity above which the radiation pressure is sufficient to support such a dust-driven wind [4]. This depends on the dust optical depth compared to the total mass in the wind, i.e. the opacity of the dust species, dust:gas mass ratio and total massloss rate. Dust only forms below a certain temperature and provided the density is high enough. On the RGB, a dust-driven wind is only possible if carbonaceous dust is formed as it has a relatively high opacity, but no carbon stars are found on the RGB (although carbon stars do exist in old globular clusters, [33]). Silicate dust can be used to drive a wind only for AGB stars significantly more luminous than the tip of the RGB [4]. This also shows that the exact evolution of a star in terms of its luminosity and especially temperature is critical for its ability to form dust and use it to drive a wind.

What happens if the pulsation and/or dust formation fail? Because these tend to be warmer, more compact stars, which are known to be chromospherically active, it is believed that chromospheric processes are likely at the origin of the driving mechanism. Instead of radiation, Alfvén (electromagnetic) or acoustic (pressure) waves are invoked. Mass-loss rates are very difficult to measure for these stars because of the sensitivity to 
the excitation/ionization balance and deviations from local thermodynamic equilibrium. A semi-empirical relation has been suggested by [22], which would lead to higher massloss rates of metal-poor stars, because they are warmer.

Observations of RGB stars do suggest that non-dusty winds may be at least as efficient as dusty winds. Modelling of optical absorption line profiles in globular cluster red giants by [19] yields mass-loss rates in excess of Reimers' Law and in agreement with emission-wing diagnostics [3] and dust-derived mass-loss rates where available [20]. Indeed, [10] show that the combined energy involved in radiation and wind is similar for chromospherically and dust-driven winds, operating in different temperature regimes but with a smooth transition zône (cf. [27]).

The luminosity and temperature are obvious measures of the radiation pressure and ability to form dust. Because evolutionary tracks do not cross, the mass is not needed as an independent parameter, $M=M(L, T)$. Both observations and hydrodynamical computations support a parameterization of the mass-loss rate of the form $\dot{M}(L, T)$, with a steep dependence on temperature but roughly proportional to luminosity [30, 31, 36] - where it must be realised that the temperature varies less than a factor two.

The mass-loss rate has also been parameterized as a function of the pulsation period, $P$ [34]. This works reasonably well at least for the sample for which it was derived, partly because $P=P(L, T)$ as it is a measure of the stellar radius, $R$, and $L=4 \pi \sigma R^{2} T^{4}$. The period sets the timescale for dust to form and grow, the amplitude of pulsation may be at least as relevant [27].

[10] suggest that irrespective of the driving mechanism, the mass-loss rate may be parameterized as a function of the surface gravity, $g$. Again, it is not obvious whether the gravity, or escape velocity, is the parameter of fundamental importance, as $g=g(L, T)$ because both the radius and mass are functions of luminosity and temperature.

Complications arise if the AGB star is in a binary system; the mass-loss rate may be enhanced equatorially, a circumbinary disc may trap ancient material, a fast(er) bipolar wind may develop, and mass transfer may enrich the surface of the companion star. In novae, mass loss from the donor indirectly contributes to explosive nucleosynthesis, and a merger of the two stars may induce an eruption.

\section{HOW DOES NUCLEOSYNTHESIS ALTER THE CONDITIONS FOR THE MASS-LOSS MECHANISM?}

Nucleosynthesis and thermal pulses affect the luminosity and temperature and thus the mass-loss rate. The evolutionary track in a Hertzsprung-Russell diagram depends on a star's initial metallicity, $Z$, helium abundance, and a changing carbon:oxygen ratio. During a thermal pulse the luminosity and temperature change in a complex manner, and depending on the $\dot{M}(L, T)$ formalism the star is normally expected to experience a brief episode of more intense mass loss. [34] predict less mass loss during the first thermal pulses, but a rapid truncation of AGB evolution, compared to Reimers' Law which allows more thermal pulses and a more balanced mass loss over the AGB evolution. If a final thermal pulse occurs in a massive AGB star after HBB ceased [29, 5], a massive carbon star is formed and fresh dust will be carbonaceous. It is unclear whether this would affect the mass-loss rate. A final thermal pulse occurring whilst on the post-AGB 


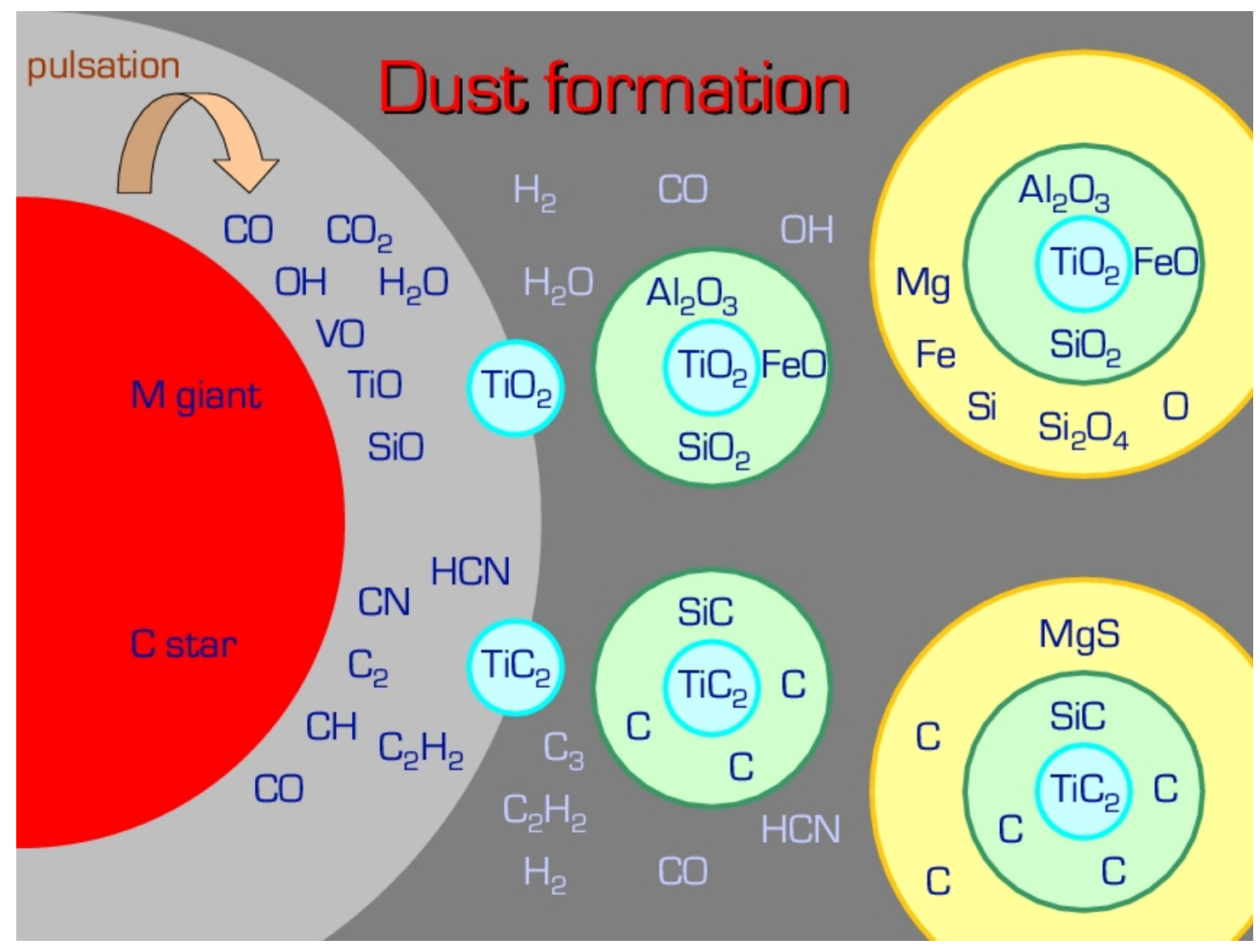

FIGURE 1. Schematic overview of the dust formation process in oxygen- (top) and carbon-dominated (bottom) circumstellar environments. Seeds involving trace chemical elements such as titanium act as condensation nuclei. First coated with highly refractory molecular species (e.g., alumina or silicon carbide), these in turn are coated with more volatile species (e.g., silicates or magnesium sulfide). The fraction of encapsulated "proper" metals such as iron may vary, affecting the transparency of the grains. Little is known about the initial nucleation and its relation to the composition of the molecular atmosphere.

track may sent the star back onto the AGB, rejuvenating its mass loss. Clearly, thermal pulses could have a great impact if they occur in a symbiotic system.

Dust formation is a complex process too. It takes place in an either oxygen-dominated or carbon-dominated environment depending what element is left after the other is exhausted in the formation of the carbon-monoxide molecule $(\mathrm{CO})$ in the cool, dense lower layers of the stellar atmosphere (Fig. 1). Molecules do not directly bind together to form solid particles, but need a nucleation seed. This is confirmed by meteoritic evidence (cf. Hoppe, these proceedings) to be based on titanium, and possibly zirconium and silicon. Neither of these are produced inside AGB stars, so their abundance is set by the initial metallicity, $Z_{\text {init }}$. The grain growth follows a sequence of decreasing condensation temperature, with aluminium-oxides and silicon-carbide ( $\mathrm{SiC}$ ) condensing before silicates and amorphous carbon (in oxygen- and carbon-rich environments, respectively). Apart from composition and size, the grain shape and degree of crystalline structure further affect the optical properties and thus the ability of the grains to drive a wind.

None of the dust species involve hydrogen or helium, which leaves it up to a minor 


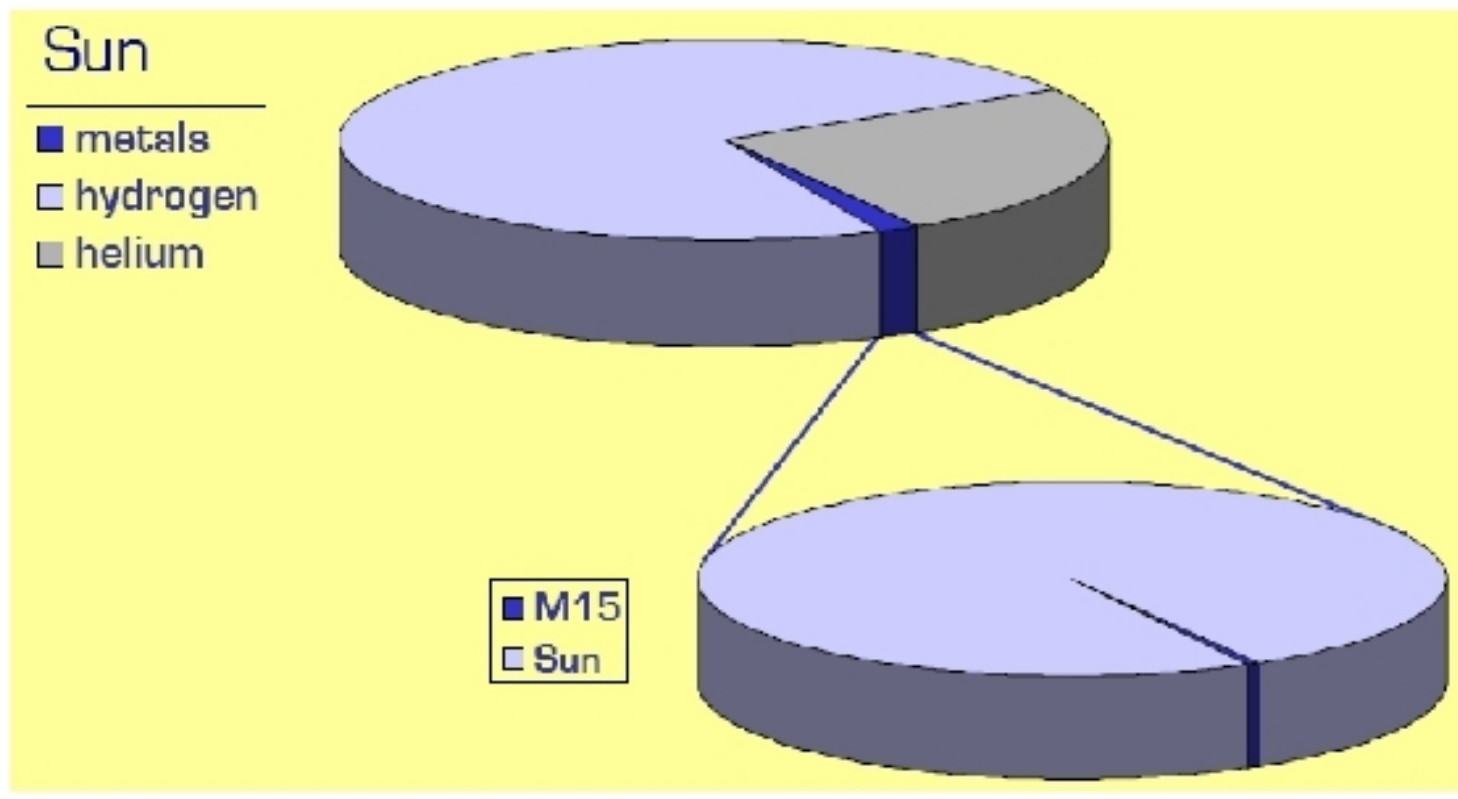

FIGURE 2. Dust is formed out of metals, which constitute only a minor fraction of any star. In metalpoor globular clusters, this fraction is truly minute yet dust is observed in at least one such cluster, M 15 .

mass fraction of the wind to provide the agents for the interception of the momentum from the radiation field. Even in the Sun it is only $\sim 1 \%$ (not all metals are used for dust formation, and $\mathrm{CO}$ takes away a large fraction of it too), but in very metal-poor environments such as in $\mathrm{M} 15$, with $Z=0.005 \mathrm{Z}_{\odot}$, dust is seen [2]. To appreciate how little mass in condensable material this is, just look at Fig. 2!

Oxygen-rich AGB stars pose a challenge: [38] reminds us of earlier findings that silicates are transparent; iron seems to be required to provide sufficient opacity where the stellar radiation peaks. [7] propose an alternative solution, if some carbonaceous dust could still be formed. Although most of the dust mass would be oxygenous, most of the opacity in the near-infrared would be due to carbonaceous dust.

Oxygenous minerals present a rich diversity, due to various admixtures of aluminium, silicon, iron, magnesium, even calcium. Mixing of the products of CNO processing could affect the amount of oxygen available, but it is generally the other elements it binds with, that are the critical component (Fig. 3). Changes in the aluminium abundance could affect the initial coating of the nucleation seeds, but it is unclear whether the later coating by silicates is affected. Hence, titanium is critical as seed, silicon for grain growth, and iron for opacity. All these scale directly with initial metallicity. This is consistent with the simple and predicted behaviour of dust-driven winds of massive AGB stars: $\dot{M}$ is insensitive to $Z_{\text {init }}$, but the dust:gas ratio $\psi \propto Z_{\text {init }}[25,26,15]$.

Metal-poor carbon stars exhibit strong molecular absorption [28, 16, 17, 32], but there is no convincing evidence for enhanced dust formation [23, 39, 14, 18]. These works show that, in sufficiently cold envelopes, magnesium-sulfide $(\mathrm{MgS})$ forms in a similar ratio to the amorphous carbon which constitutes the bulk of carbonaceous dust. This suggests that if $\mathrm{MgS}$ is depleted in metal-poor winds because of lower 


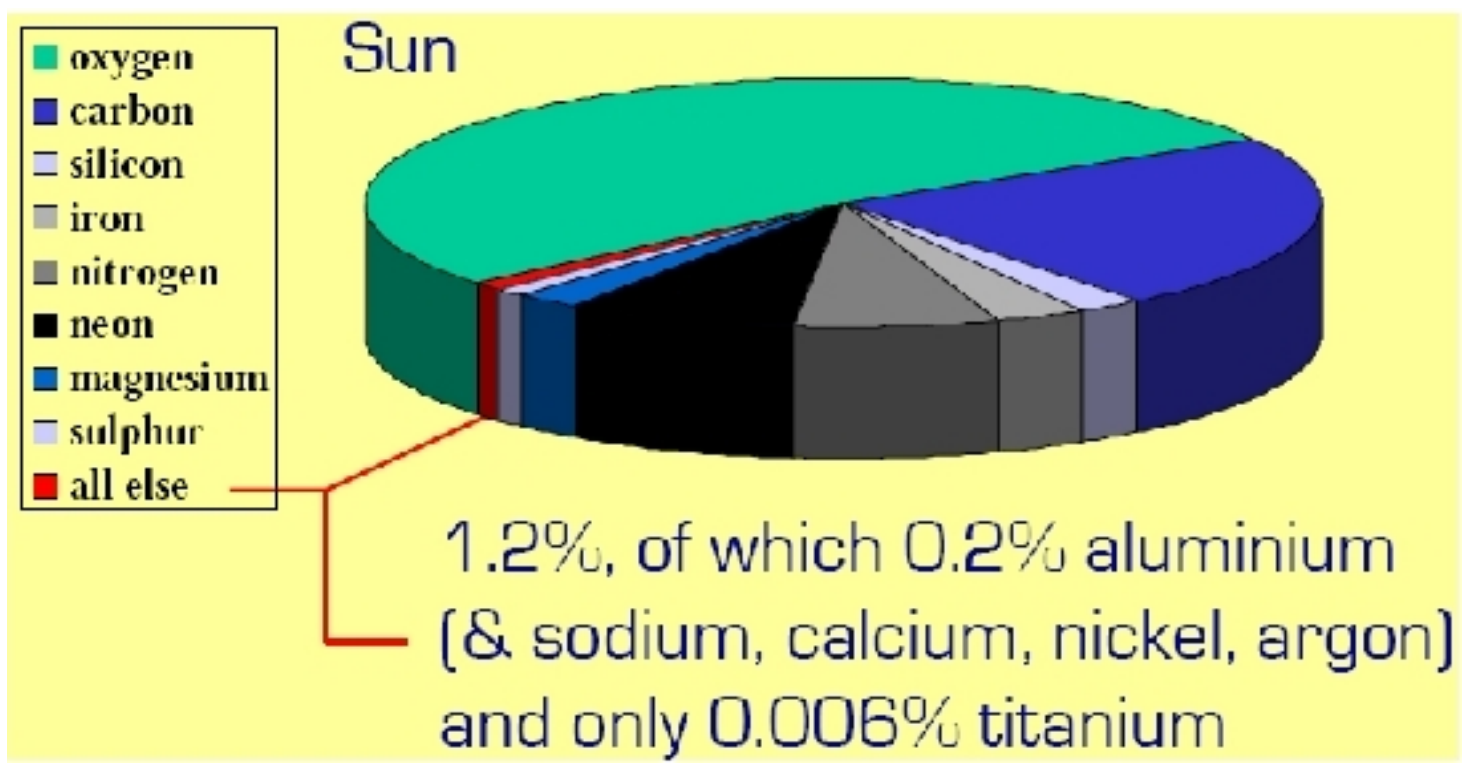

FIGURE 3. Approximate fractions of the metals in the Sun, of various elements. Oxygen and/or carbon, which form the basis of most of the circumstellar dust produced in AGB winds, are always abundant. Important constituents of certain dust species, iron, silicon, magnesium and aluminium are much less abundant and thus limit grain growth. As a nucleation seed, titanium is a key element but extremely rare.

abundances of magnesium and sulphur, amorphous carbon must be depleted too. SiC is more complicated to interpret as it may be coated by amorphous carbon.

In carbon star winds too, the nucleation seeds depend on titanium and similar elements. The strong molecular bands especially of the slightly larger molecules such as acetylene and $\mathrm{C}_{3}$ suggest that the density of molecules may not be larger, but they may be found throughout a larger part of the wind [32]. The grain growth may thus not be any different. Indeed, the observed spectral energy distributions are consistent with $\psi \propto Z_{\text {init }}$ and $\dot{M}$ insensitive to $Z_{\text {init }}[25,26$, and this is also supported by considerations of the molecular mass-loss rate [17], the availability of silicon in gas phase and SiC [18], and the lower dust production seen in magellanic PNe [24].

$\mathrm{S}$ stars, which have a carbon:oxygen ratio approaching unity, are a relatively unimportant contributor to chemical enrichment and unimportant phase for the star itself, because the mass-loss rate is not particularly high and the phase lasts very few thermal pulse cycles. They may teach us something about dust formation and growth, though, when oxygen or carbon have become critical elements in the chain of events.

\section{ACKNOWLEDGMENTS}

I would like to thank Maurizio Busso for inviting me to the workshop to talk about AGB mass loss, all the participants for a very interesting and joyous time together, and Joana for putting up admirably with my busy travel diary. This talk was presented on 26 October 2007, which was also the "Wear it Pink" awareness day of the british Breast Cancer Campaign, to which this talk is dedicated. Oh yes, and the food was very good! 


\section{REFERENCES}

1. G. H. Bowen, \& L. A. Willson, ApJ, 375, L53 (1991).

2. M. L. Boyer, C. E. Woodward, J. Th. van Loon, K. D. Gordon, A. Evans, R. D. Gehrz, L. A. Helton, \& E. F. Polomski, $A J, \mathbf{1 3 2}, 1415$ (2006).

3. J. G. Cohen, ApJ, 203, L127 (1976).

4. A. S. Ferrarotti, \& H.-P. Gail, $A \& A, 447,553$ (2006).

5. C. A. Frost, R. C. Cannon, J. C. Lattanzio, P. R. Wood, \& M. Forestini, A\&A, 332, L17 (1998).

6. B. M. S. Hansen, ApJ, 635, 522 (2005).

7. S. Höfner, \& A. Andersen, $A \& A, \mathbf{4 6 5}$, L39 (2007).

8. D. C. Jackson, E. D. Skillman, R. D. Gehrz, E. Polomski, C. E. \& Woodward, ApJ, 656, 818 (2007a).

9. D. C. Jackson, E. D. Skillman, R. D. Gehrz, E. Polomski, \& C. E. Woodward, ApJ, 667, 891 (2007b).

10. P. G. Judge, \& R. E. Stencel, ApJ, 371, 357 (1991).

11. J. S. Kalirai, P. Bergeron, B. M. S. Hansen, D. D. Kelson, D. B. Reitzel, R. M. Rich, \& H. B. Richer, ApJ, 671, 748 (2007).

12. A. I. Karakas, M. A. Lugaro, M. Wiescher, J. Görres, \& C. Ugalde, ApJ, 643, 471 (2006).

13. E. Lagadec, et al., MNRAS, 376, 1270 (2007a).

14. E. Lagadec, A. A. Zijlstra, M. Matsuura, J. W. Menzies, J. Th. van Loon, \& P. A. Whitelock, MNRAS, in press (2007b).

15. J. R. Marshall, J. Th. van Loon, M. Matsuura, P. R. Wood, A. A. Zijlstra, \& P. A. Whitelock, MNRAS, 355, 1348 (2004).

16. M Matsuura, et al., $A \& A, 434,691$ (2005).

17. Matsuura, M., et al., MNRAS, 371, 415 (2006).

18. M. Matsuura, et al., MNRAS, 382, 1889 (2007).

19. I. McDonald, \& J. Th. van Loon, A\&A, 476, 1261 (2007).

20. L. Origlia, F. R. Ferraro, F. Fusi Pecci, \& R. T. Rood, ApJ, 571, 458 (2002).

21. D. Reimers, Societé Royale des Sciences de Liège, Memoires, 8, p369 (1975).

22. K.-P. Schröder, \& M. Cuntz, ApJ, 630, L73 (2005).

23. G. C. Sloan, K. E. Kraemer, M. Matsuura, P. R. Wood, S. D. Price, \& M. P. Egan, ApJ, 645, 1118 (2006).

24. L. Stanghellini, P. García-Lario, D. A. García-Hernández, J. V. Perea-Calderón, J. E. Davies, A. Manchado, E. Villaver, \& R. A. Shaw, ApJ, in press (2007).

25. J. Th. van Loon, $A \& A$ 354, 125 (2000).

26. J. Th. van Loon, in: Stellar Evolution at Low Metallicity, eds. H. J. G. L. M. Lamers, N. Langer, T. Nugis, \& K. Annuk, ASPC, 353, p211 (2006).

27. J. Th. van Loon, in: XXI Century Challenges for Stellar Evolution, eds. S. Cassisi \& M. Salaris, MemSAIt, 79/2 (2008).

28. J. Th. van Loon, A. A. Zijlstra, \& M. A. T. Groenewegen, A\&A, 346, 805 (1999).

29. J. Th. van Loon, et al., $A \& A, 329,169$ (1998).

30. J. Th. van Loon, M. A. T. Groenewegen, A. de Koter, N. R. Trams, L. B. F. M. Waters, A. A. Zijlstra, P. A. Whitelock, \& C. Loup, A\&A, 351, 559 (1999).

31. J. Th. van Loon, M.-R. L. Cioni, A. A. Zijlstra, \& C. Loup, A\&A, 438, 273 (2005).

32. J. Th. van Loon, J. R. Marshall, M. Cohen, M. Matsuura, P. R. Wood, I. Yamamura, \& A. A. Zijlstra, $A \& A, 447,971(2006)$

33. J. Th. van Loon, F. van Leeuwen, B. Smalley, A. W. Smith, N. A. Lyons, I. McDonald, M. L. \& Boyer, MNRAS, 382, 1353 (2007).

34. E. Vassiliadis, \& P.R. Wood, ApJ, 413, 641 (1993).

35. E. Villaver, L. Stanghellini, R. A. \& Shaw, ApJ, 656, 831 (2007).

36. A. Wachter, K.-P. Schröder, J. M. Winters, T. U. Arndt, \& E. Sedlmayr, A\&A, 384, 452 (2002).

37. K. A. Williams, in: $15^{\text {th }}$ European Workshop on White Dwarfs, eds. R. Napiwotzki \& M. Burleigh, $A S P C$, 372, p85 (2007).

38. P. Woitke, $A \& A, \mathbf{4 6 0}$, L9 (2006).

39. A. A. Zijlstra, et al., MNRAS, 370, 1961 (2006). 